\title{
RESEARCH
}

\section{ATM deficiency promotes progression of CRPC by enhancing Warburg effect}

\author{
Lingfan Xu1,2, Enze Ma33, Tao Zeng2,4, Ruya Zhao5, Yulei Tao², Xufeng Chen², Jeff Groth2, Chaozhao Liang1, \\ Hailiang $\mathrm{Hu}^{2,6}$ and Jiaoti Huang2,6,7 \\ 'Department of Urology, The First Affiliated Hospital of Anhui Medical University, Hefei, China \\ 2Department of Pathology, Duke University School of Medicine, Durham, North Carolina, USA \\ 3Department of Neuroscience, Duke University, Durham, North Carolina, USA \\ ${ }^{4}$ Department of Urology, Jiangxi Province People's Hospital, Nanchang, China \\ 5Department of Dermatology, Duke School of Medicine, Durham, North Carolina, USA \\ ${ }^{6}$ Duke Cancer Institute, Duke University School of Medicine, Durham, North Carolina, USA \\ 7Department of Pharmacology and Cancer Biology, Duke University School of Medicine, Durham, North Carolina, USA
}

Correspondence should be addressed to $\mathrm{H}$ Hu or C Liang or J Huang:

hailiang.hu@duke.edu or liang_chaozhao@163.com or jiaoti.huang@duke.edu

\begin{abstract}
ATM is a well-known master regulator of double strand break (DSB) DNA repair and the defective DNA repair has been therapeutically exploited to develop PARP inhibitors based on the synthetic lethality strategy. ATM mutation is found with increased prevalence in advanced metastatic castration-resistant prostate cancer (mCRPC). However, the molecular mechanisms underlying ATM mutation-driving disease progression are still largely unknown. Here, we report that ATM mutation contributes to the CRPC progression through a metabolic rather than DNA repair mechanism. We showed that ATM deficiency generated by CRISPR/Cas9 editing promoted CRPC cell proliferation and xenograft tumor growth. ATM deficiency altered cellular metabolism and enhanced Warburg effect in CRPC cells. We demonstrated that ATM deficiency shunted the glucose flux to aerobic glycolysis by upregulating LDHA expression, which generated more lactate and produced less mitochondrial ROS to promote CRPC cell growth. Inhibition of LDHA by siRNA or inhibitor FX11 generated less lactate and accumulated more ROS in ATM-deficient CRPC cells and therefore potentiated the cell death of ATM-deficient CRPC cells. These findings suggest a new therapeutic strategy for ATM-mutant CRPC patients by targeting LDHA-mediated glycolysis metabolism, which might be effective for the PARP inhibitor resistant mCRPC tumors.
\end{abstract} Key Words
- ATM
- glycolysis
- LDHA
- castration-resistant

\section{Introduction}

ATM (Ataxia Telangiectasia Mutated) mutation that usually leads to loss of function is found in many types of cancer, ranging from hematological malignancies to solid tumors (Choi et al. 2016). ATM protein kinase is best known as a chief guardian to protect genomic integrity through its DNA repair functions (Shiloh 2014).
In regard to prostate cancer, ATM mutation is identified in $\sim 2 \%$ of localized, non-indolent prostate cancer (Fraser et al. 2017). In advanced metastatic castration-resistant prostate cancer (mCRPC), the prevalence of ATM mutation increases to 5-11\% (Beltran et al. 2013, Robinson et al. 2015, Abida et al. 2017). Notably, ATM mutation
(C) 2019 Society for Endocrinology Published by Bioscientifica Ltd. Printed in Great Britain 
is the second largest group, next to BRCA2 mutation, of DNA-repair gene (DRG) mutation that is found $>20 \%$ of mCRPC (Robinson et al. 2015). The identification of DRG mutation status in prostate cancer (most commonly BRCA2 and ATM) provides us not only the prognostic biomarkers to predict disease progression, but also new druggable targets for the treatment of mCRPC. Germline mutations of BRCA1/2 and ATM have been shown to distinguish the risk for lethal and indolent prostate cancer and are associated with shorter survival time ( $\mathrm{Na}$ et al. 2017). The clinical impact of DRG mutation on CRPC patients' response to next-generation hormonal therapy (abiraterone, enzalutamide) remains uncertain as two studies show that CRPC patients with DRG mutations have worse response to abiraterone and enzalutamide (Annala et al. 2018, Antonarakis et al. 2018), whereas another two studies demonstrate that DRG mutationbearing CRPC patients have superior response to enzalutamide and abiraterone (Antonarakis et al. 2018, Hussain et al. 2018). Despite the response discrepancy, the mutations of BRCA1/2 and ATM are known as highly promising prognostic biomarkers to predict the therapeutic response of CRPC patients to androgen receptor (AR)-targeted therapies. On the other hand, DRG mutations in mCRPC patients can be therapeutically exploited with PARP inhibitor based on the synthetic lethality strategy (Mateo et al. 2015).

In addition to its hierarchical regulatory role in DNA repair, ATM has been shown to play a critical role in regulating cellular metabolism (Ambrose \& Gatti 2013, Shiloh \& Ziv 2013). Cancer cells can rewire their metabolic pathways to meet their needs for malignant transformation (Cairns et al. 2011). A well-known metabolic phenotype is Warburg effect, which refers to the increased glucose consumption and lactate production in cancer cells even under normal oxygen concentration (Warburg 1956). The functions of Warburg effect remain unclear, but it is proposed to promote tumor progression through several mechanisms, such as increasing access to a limited energy source, enhancing immune cell evasion and allowing for signal transduction through ROS or chromatin modulation (Liberti \& Locasale 2016). ATMdeficient human lymphoblastoid cells and neural stem cells have been shown to have intrinsic mitochondrial dysfunctions with defective respiration capacity (Ambrose et al. 2007, Lee et al. 2013). A recent study demonstrated that ATM inactivation decreases p53 and upregulates c-Myc to enhance glucose and glutamine consumption, which functions to bypass the nutrient deficiency-induced senescence (Aird et al. 2015). These studies suggest that
ATM function in metabolism is different from its classic role in DNA repair.

In this study, we report that ATM deficiency leads to enhanced Warburg effect in CRPC cells and as a consequence promotes the progression of CRPC. We demonstrated that ATM deficiency further diverted the glucose flux from mitochondria-mediated oxidative phosphorylation (OXPHOS) pathway to aerobic glycolysis by upregulating lactate dehydrogenase A (LDHA) expression. Inhibition of LDHA by siRNA or inhibitor increased ROS level in cells and suppressed the cell growth of ATM-deficient CRPC cells, which suggests a potential alternative therapeutic strategy for ATM mutation-bearing CRPC patients.

\section{Materials and methods}

\section{Cell culture}

C4-2 and CWRR1 human prostate cancer cell lines were obtained from American type Culture Collection (ATCC). All experiments were performed using cells that were $<10$ passages. C4-2 and CWRR1 cells were cultured in RPMI-1640 medium (ATCC) supplemented with 10\% fetal bovine serum (FBS; Corning) and 100 units $/ \mathrm{mL}$ penicillin/streptomycin (Gibco) in a $5 \%$ (vol/vol) $\mathrm{CO}_{2}$ and $95 \%$ (vol/vol) air incubator at $37^{\circ} \mathrm{C}$.

\section{Colony formation assay}

$1 \times 10^{4}$ Cells were seeded into $6 \mathrm{~cm}$ dishes and grown for up to 14 days for colony formation. Cell culture media was refreshed every 3 days and colonies were fixed with cold methanol and then stained with $1 \%$ crystal violet. The number of colonies was counted and imaged with a VersaDoc Imaging System (BioRad).

\section{CRISPR/Cas9-mediated ATM knockout}

To establish ATM knockout stable cell line, we used the CRIPR/Cas9 technology. Single guided RNA (sgRNA) sequences were generated by CRISPR design tool (crispr. mit.edu). sgRNA sequences for ATM are available upon request. Annealed double-stranded sgRNA oligos were ligated to the lentiCRISPR vector 2 (deposited by Dr Feng Zhang to Addgene, Cambridge, MA, USA) at a ribonucleoprotein complex, which expressed both Cas9 and sgRNA. To produce infectious transgenic lentivirus, the transfer plasmid was transfected into 293T cells together with packaging plasmid and envelope plasmid. 
After the change in medium and a brief incubation period, supernatant containing the virus was collected and centrifuged to concentrate virus. Subsequently, cells were selected by puromycin for more than 14 days. Population that showed no target protein expression was confirmed by western blot.

\section{RNA interference}

siRNAs targeting human LDHA (ON-TARGETplus SMARTpool: L-008201-00-0005) and ON-TARGETplus Nontargeting pool were purchased from Dharmacon. siRNAs targeting human c-Myc (sc-29226) were purchased from Santa Cruz. 105/well C4-2/Ctrl (CWRR1/Ctrl) or C4-2/ATM-KO (CWRR1/ATM-KO) cells were seeded in sixwell plate. After $24 \mathrm{~h}, 30 \mathrm{pmol}$ siLDHA/sic-Myc or siCtrl was transfected into cells using Lipofectamine RNAiMAX (Invitrogen) in Opti-MEM reduced serum medium (Gibco) for $24 \mathrm{~h}$.

\section{Cell viability assays}

The MTS assay (Biovision) was employed to determine cell viability. 1000/well cells were seeded in 96-well plate. $10 \mu \mathrm{L} /$ well MTS Reagent was added into each well and incubated for $1 \mathrm{~h}$ at each time point. Absorbance was measured at $490 \mathrm{~nm}$ using SpectraMax M3 reader with SoftMax Pro 6 software for data acquisition and analysis. For cells treated with siRNAs, 5 pmol siLDHA or siCtrl was transfected into each well and cell viability was measured at each time point. For cells treated with FX11 (Millipore), $3 \mu \mathrm{M}$ FX11 was added in culture medium followed by cell viability measurement. For $\mathrm{IC}_{50}$ determination of FX11, cells were treated by a series of concentration for $72 \mathrm{~h}$. $\mathrm{IC}_{50}$ value was calculated by GraphPad Prism software.

\section{Animal study}

The animal experiment protocol (A092-16-04) was reviewed and approved by the Duke Institutional Animal Care and Use Committee. Immunocompromised NSG mice were purchased from Jackson Laboratories. $10^{7}$ C4-2/Ctrl or C4-2/ATM-KO cells were resuspended in $100 \mu \mathrm{L}$ of saline with $50 \%$ Matrigel (Corning) and injected subcutaneously into both sides of flank regions of 4- to 6-week-old male NSG mice. When tumor volume reached a size of $100 \mathrm{~mm}^{3}$ which was set as Day 0, tumors were measured twice a week and tumor volumes were determined by caliper measurement of tumor length (L) Printed in Great Britain and width (W) according to the formula $\left(\mathrm{L} \times \mathrm{W}^{2}\right) / 2$. For FX11 in vivo experiment, when tumor volume reached $200 \mathrm{~mm}^{3}$, C4-2/Ctrl and C4-2/ATM-KO grafted mice were divided into two groups randomly. Each group of mice was injected with either control $2 \%$ (vol/vol) DMSO or $50 \mu \mathrm{g}$ of FX11 intraperitoneally every other day. Tumor volumes were measured every 4 days.

\section{Immunohistochemical staining and quick-score system}

Immunohistochemical staining (IHC) was performed as described in previous study (Park et al. 2017). Xenograft tumor sections were deparaffinized and rehydrated. Endogenous peroxidase activity was blocked with 3\% hydrogen peroxide in methanol. Heat-induced antigen retrieval was carried out for all sections in $0.01 \mathrm{M}$ citrate buffer, $\mathrm{pH} 6.0$, using a steamer at $95^{\circ} \mathrm{C}$. Primary antibodies were diluted with PBS containing $1 \%$ BSA to an optimal concentration and applied to the sections overnight. Slides were then incubated with rabbit antimouse or goat anti-rabbit IgG/HRP second antibody for $45 \mathrm{~min}$. Diaminobenzidine (DAB) was then applied for $10 \mathrm{~min}$. The sections were counterstained with hematoxylin, dehydrated, coverslipped and visualized. Primary antibodies used in this study are ant-CD31 (Abcam, Ab28364, 1:100), anti-Ki67 (ThermoFisher, RM-9106, 1:300) and anti-Snail (Abcam, Ab85936, 1:600). Quick-score was calculated as reported (Park et al. 2017), the intensity of staining is multiplied by the percentage of staining to derive a composite score (a range from 0 to 300$)$.

\section{Metabolite extraction and mass spectrometry}

As described previously (Pan et al. 2016), 105/well cells were plated in six-well plate. Twenty-four hours later, medium was replaced freshly as equal volume $(2 \mathrm{~mL})$ per well and cells were incubated for another $24 \mathrm{~h}$. Next, medium was aspirated and $80 \%$ (vol/vol) HPLC grade methanol/water was added into each well. Plates were placed on dry ice immediately and then transferred in a $-80^{\circ} \mathrm{C}$ freezer for $15 \mathrm{~min}$ for further enzyme inactivation. Cells were then scraped into extraction solvent. After centrifuge for $10 \mathrm{~min}$, supernatant was transferred into a new Eppendorf tube. A speed vacuum was used to dry the samples at room temperature, and then the dry pellets were sent to LOCASALE LAB (Duke University) for further liquid chromatography mass spectrometry analysis (LC-MS). Data were normalized to cell numbers. 


\section{${ }^{13} \mathrm{C}$ isotope tracing assay}

$10^{5} /$ well cells were seeded in six-well plate in $2 \mathrm{~mL}$ complete medium. After overnight incubation, medium was removed and replaced by exact $2 \mathrm{~mL}$ fresh glucosedeprived media (Gibco 11966) containing $25 \mathrm{mmol} / \mathrm{L}$ uniformly ${ }^{13} \mathrm{C}$-labeled glucose isotope (Cambridge). After 24-h incubation, metabolites were extracted and sent for further LC-MS analysis.

\section{Glucose consumption assay}

Glucose concentration in culture medium was determined by Glucose Colorimetric/Fluorometric Assay Kit (Biovision) following the manufacturer's protocol. Glucose consumption was calculated as measured value subtracted by original concentration.

\section{Lactate secretion assay}

The lactate level in the medium was analyzed by Lactate Colorimetric/Fluorometric Assay Kit (Biovision). Ten microliters of sample were added into $40 \mu \mathrm{L}$ lactate assay buffer and then $50 \mu \mathrm{L}$ reaction mix were added into each diluted sample. The reaction was incubated for $30 \mathrm{~min}$ at room temperature while being protected from light. Absorbance at $\mathrm{OD}_{570 \mathrm{~nm}}$ was measured by SpectraMax M3 reader. All assays were performed in triplicate.

\section{RNA extraction and quantitative real-time PCR}

Total RNA was extracted using RNeasy mini kits (Qiagen). cDNA synthesis was performed by PrimeScript RT Master Mix (Takara). Quantitative real-time PCR (qRT-PCR) was applied on ABI 7500 (Applied Biosystems) using SYBR Green PCR Master Mix (Quanta Biosciences). Actin expression assay was used as an internal control. All qPCRs were determined in triplicate. Sequences of primers use in study are available upon request.

\section{Immunoblotting}

Total protein was collected by lysing adherent cells with RIPA buffer (Sigma) supplemented with phosphatase and protease inhibitor cocktail (Thermo Fisher). For nuclear protein extraction, nuclear lysates were isolated with an NE-PER Nuclear and Cytoplasmic Extraction Reagents Kit (Thermo) with phosphatase and protease inhibitor cocktail contained. Protein concentration was measured using the Bradford reagent (Bio-Rad). Equal amount of protein was loaded for immunoblotting. After electrophoresis, the protein was transferred to PVDF transfer membrane followed by 5\% non-fat milk blocking buffer and incubated overnight with primary antibodies. After washing with TBST (TBS with $0.1 \%$ Tween), antimouse or anti-rabbit second antibodies conjugated with HRP-conjugated were used to probe the membranes. Samples were developed by Chemiluminescent Substrate (Thermo Fisher) and exposed by Odyssey Imaging Systems (LI-COR). Densitometry was performed using ImageJ software (National Institutes of Health) and samples were normalized to internal controls. Primary antibodies used in this study are anti-ATM (Novus, NB100-104), anti-p84 (Thermo Fisher, PA4-27816), anti-LDHA (Cell Signaling, 2012S), anti-c-Myc (Abcam, 32072) and anti-GAPDH (Abcam, Ab9484).

\section{ROS measurement}

As previously described(Le et al. 2010), intracellular ROS production was determined by staining cells with $10 \mu \mathrm{M}$ 5-(and-6)-carboxy-2',7' -dichlorodihydrofluorescein diacetate (carboxy-H2DCFDA, Thermo Fisher) according to the manufacturer's instructions. $10^{5}$ cells/well (six-well plate) were treated with either $3 \mu \mathrm{M}$ FX11 for $72 \mathrm{~h}$ or $30 \mathrm{pmol}$ siLDHA for $24 \mathrm{~h}$. Stained cells were analyzed by FACScan flow cytometers at excitation and emission wavelengths of 485 and $520 \mathrm{~nm}$, respectively.

\section{Statistical analysis}

All experiments were performed at least three times and data were presented as the mean \pm standard deviation (s.D.). Statistically significant differences between two groups were assessed using a two-tail paired $t$-test. $P<0.05$ was considered significant. All statistical analyses were performed using GraphPad Prism software and Microsoft Excel. Principal component analysis (PCA) and enrichment of metabolite pathways were performed by MetaboAnalyst online software (http://www.metaboanalyst.ca/).

\section{Results}

\section{ATM deficiency promotes CRPC progression}

ATM mutations including missense mutations usually lead to loss of or reduced function of ATM kinase activity (Hu \& Gatti 2008). To address whether ATM mutation affects CRPC progression, we established ATM knockout (ATM-KO) and corresponding control (Ctrl) stable cell (c) 2019 Society for Endocrinology Published by Bioscientifica Ltd. Printed in Great Britain 
lines by CRISPR/Cas9 editing for C4-2 and CWRR1, two CRPC cell lines. ATM knockout unexpectedly increased cell proliferation and colony formation in C4-2 cells as shown by the cell growth curve and colony formation assay (Fig. 1A). A similar result of cell proliferation and colony formation was observed for CWRR1 cells (Fig. 1B), suggesting that ATM deficiency promotes CRPC cell growth, at least in the two examined CRPC cell lines. To confirm this observation in vivo, we subcutaneously injected C4-2/Ctrl and C4-2/ATM-KO cells into immunodeficient SCID/NSG mice and monitored the xenograft tumor formation. As shown in Fig. 1C, ATM knockout significantly promoted the xenograft tumor growth, which is consistent with the in vitro proliferation and colony formation results. The xenograft tumors were then dissected at the end of the in vivo experiment and subjected to immunohistochemistry (IHC) staining analysis. ATM-KO tumors were obviously larger and heavier than control tumors (Fig. 1D and E). The hematoxylin and eosin (H\&E) staining showed that ATM-KO tumors had less cell necrosis compared to that of control tumors (Fig. 1F), suggestive of increased cell proliferation and decreased cell death in ATM-KO tumors. The increased proliferation was further validated by a robust Ki-67 (a proliferation
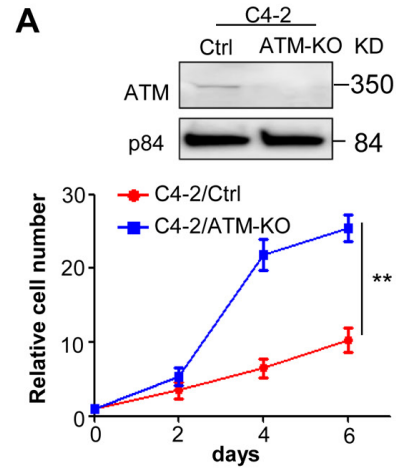

C

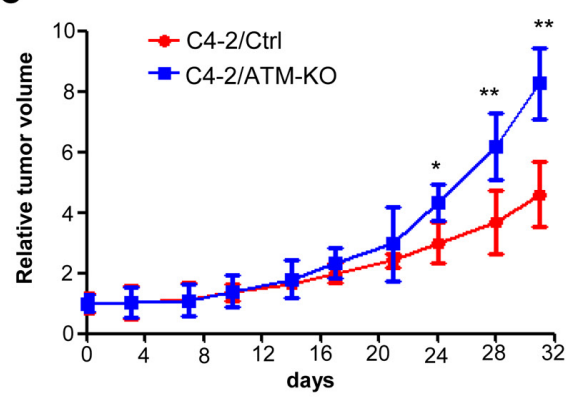

D

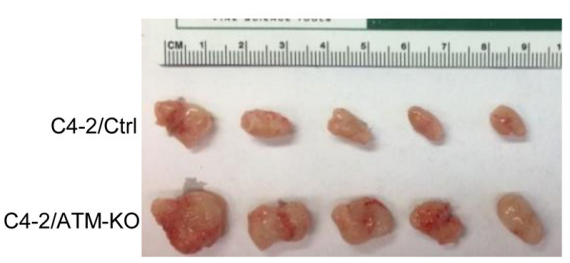

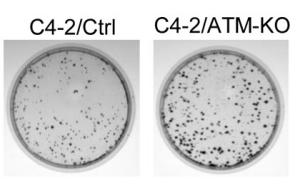

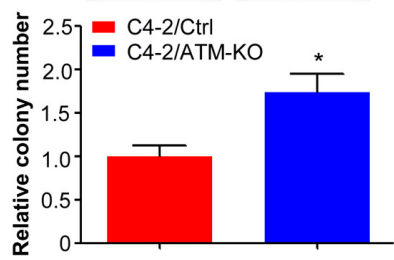

B

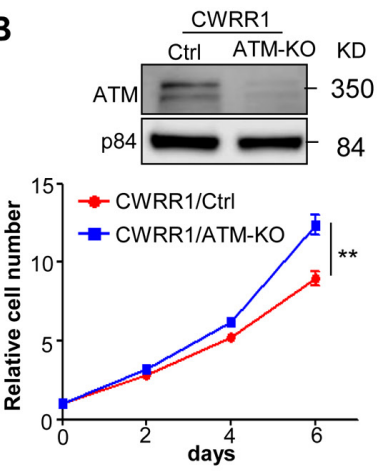

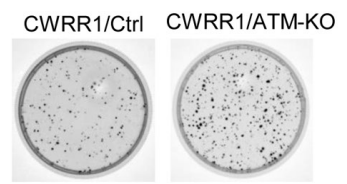

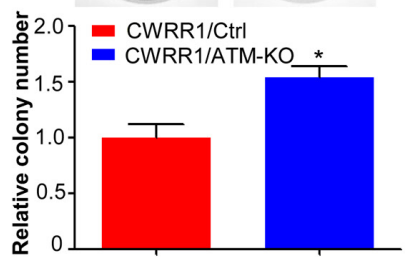

$\mathbf{F}$

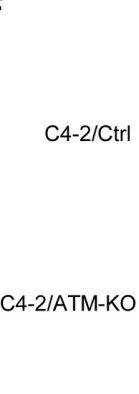

E

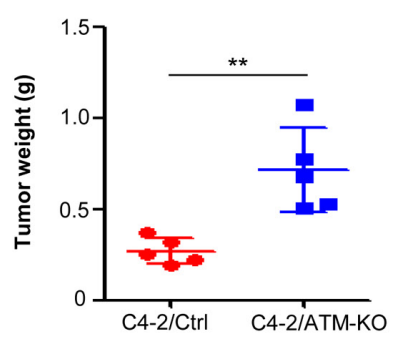

G

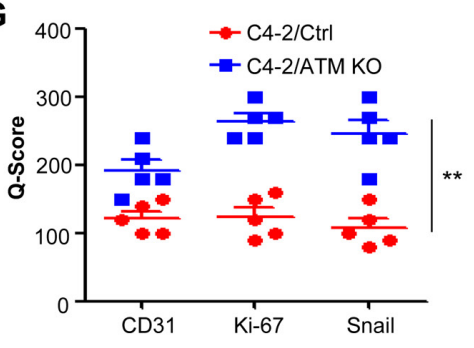

Figure 1

ATM deficiency promotes CRPC progression. (A and B) Cell proliferation (left panel) and colony formation (right panel) of C4-2/Ctrl vs C4-2/ATM-KO cells and CWRR1/Ctrl vs CWRR1/ATM-KO cells generated by CRISPR/Cas9 technology. Top: nuclear lysates were extracted and ATM expression was determined by Western blot. p84 was used as a loading control. Bottom: cell proliferation was measured by MTS assay at each indicated time points and normalized to Day 0 ( $n=3$ replicates). Colony formation assay was performed for C4-2/Ctrl vs C4-2/ATM-KO cells and CWRR1/Ctrl vs CWRR1/ATM-KO cells. Top: representative images for formed colonies. Bottom: graphs showing the quantifications of colonies formed ( $n=3$ replicates). (C) Xenograft tumor growth of C4-2 stable cell lines generated in (A). Tumor volumes were measured twice a week and normalized to Day 0 ( $n=5$ mice per group). (D) Representative tumors in each group. (E) The weight of xenograft tumors obtained at the end of animal study ( $n=5$ mice per group). (F) Representative images of H\&E and IHC analysis of CD31, Ki-67 and Snail in xenografts obtained from (E). Magnification: 200×. (G) A plot of Quick-score for IHC staining in (F). All data are depicted as mean \pm S.D. ${ }^{*} P<0.05$ and $* \star P<0.01$ by two-tailed Student's $t$-test. 
marker) staining in ATM-KO tumors (Fig. 1F and G). In addition, ATM-KO tumors showed a strong expression in both vasculature and epithelial-to-mesenchymal transition (EMT), determined by CD31 and Snail staining (Fig. $1 \mathrm{~F}$ and $\mathrm{G}$ ), indicating that ATM-KO CRPC tumors are more aggressive than control tumors. Taken together, we showed that ATM deficiency promotes CRPC progression both in vitro and in vivo.

\section{ATM deficiency alters cellular metabolism and enhances Warburg effect in CRPC cells}

Cellular metabolic reprogramming has been shown to support their rapid proliferation and sustainable growth of cancer cells (Pavlova \& Thompson 2016). To test whether ATM deficiency-induced CRPC progression is caused by alteration of cellular metabolism, we conducted a metabolite profiling for C4-2/Ctrl and C4-2/ATM-KO cells. Notably, ATM deficiency resulted in a significant change of global metabolites in C4-2 cells (Fig. 2A and
Supplementary Table 1, see section on supplementary data given at the end of this article). This observation is supported by a principal component analysis in which C4-2/ATM-KO cells were segregated from C4-2/Ctrl cells on metabolite profiles (Fig. 2B). We further analyzed the metabolic pathways associated with the changed metabolites and found that ATM deficiency significantly altered a large number of metabolism pathways, especially glucose-related energy pathways (Fig. 2C). It is well known that cancer cells produce energy to support their growth through aerobic glycolysis instead of mitochondriamediated OXPHOS (Warburg effect). To investigate whether ATM deficiency affects Warburg effect in CRPC cells, we compared the levels of intermediate metabolites during the aerobic glycolysis pathway from the profiling data. All glycolytic intermediates, except pyruvate, were shown significantly higher levels in C4-2/ATM-KO cells compared to that in C4-2/Ctrl cells (Fig. 2D). In agreement with the profiling data, ATM deficiency led to an increase in both glucose consumption and lactate
A

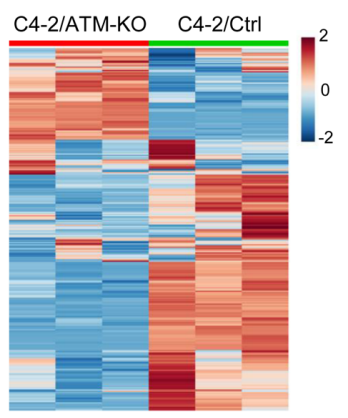

D

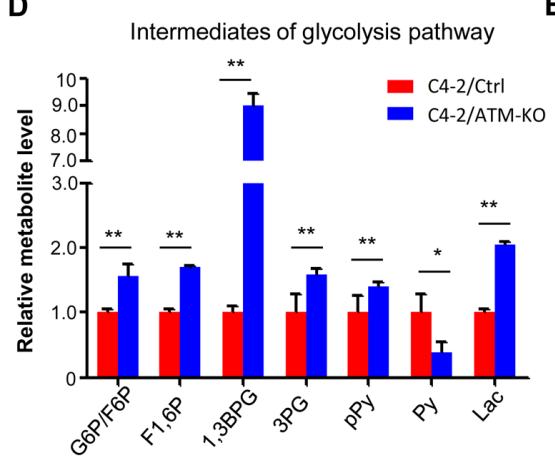

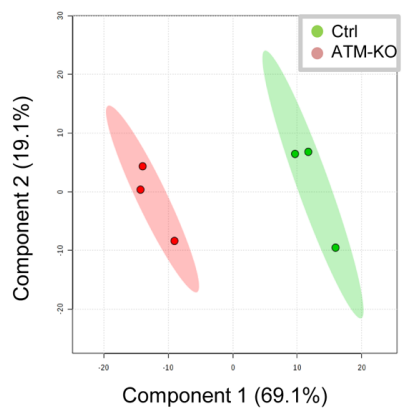

E

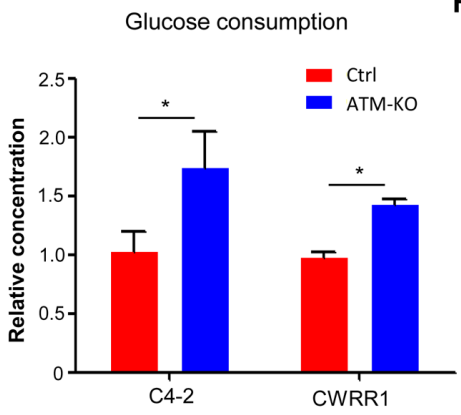

C

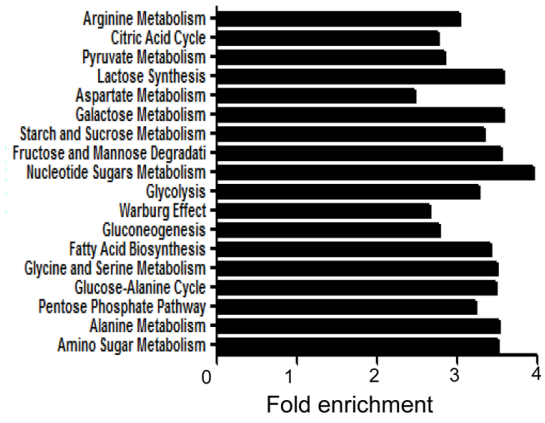

$\mathbf{F}$

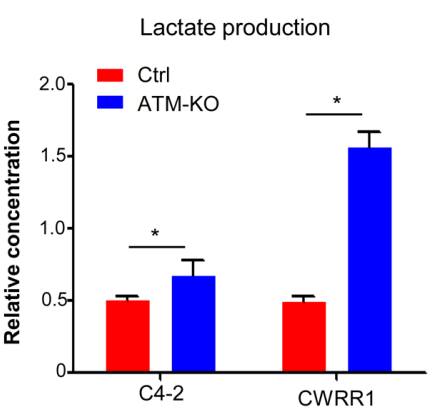

\section{Figure 2}

ATM deficiency leads to an enhanced glycolytic utilization in CRPC cells. (A) Heat map to show levels of global metabolites in C4-2/Ctrl and C4-2/ATM-KO cells ( $n=3$ biological replicates). (B) Principal component analysis (PCA) of metabolic distributions in the indicated cell lines. (C) Enriched glucose-related metabolite pathways in C4-2/ATM-KO cells. Statistically significant differences were assessed by MetaboAnalyst software as indicated in the Materials and Methods. (D) LC-MS analysis of cell extractions to compare the levels of glycolytic intermediates between C4-2/Ctrl and C4-2/ATM-KO cells ( $n=3$ ). 1,3BPG, 1,3-bisphosphoglycerate; 3PG, 3-phosphoglycerate; F1,6P, fructose 1,6-bisphosphate; F6P, fructose 6-phosphate; G6P, glucose 6-phosphate; Lac, lactate; pPy, phosphoenolpyruvate; Py, pyruvate. (E and F) Glucose consumption and lactate production in paired C4-2/Ctrl and C4-2/ATM-KO, CWRR1/Ctrl and CWRR1/ATM-KO cells. Glucose consumption was calculated as indicated in the Materials and Methods. Results are normalized to the control group $(n=3)$. All data are depicted as mean \pm S.D. ${ }^{*} P<0.05$ and ${ }^{*} P P<0.01$ by two-tailed Student's $t$-test. 
production in C4-2 cells (Fig. 2E and F). A similar result of increased glucose consumption and lactate production was observed in another ATM-deficient CRPC CWRR1 cells (Fig. 2E and F). Therefore ATM-deficient CRPC cells have an altered metabolism profile with enhanced aerobic glycolysis, which suggests that it is highly likely that ATM deficiency promotes CRPC progression through enhanced aerobic glycolysis.

\section{ATM deficiency detours glucose flux into aerobic glycolysis in CRPC cells}

To further validate the enhanced Warburg effect in ATMdeficient CRPC cells, we used uniformly ${ }^{13}$ C-labeled glucose ([U-13C]-Glc) to trace the glucose influx in C4-2/Ctrl and C4-2/ATM-KO cells. Three different glucose-derived metabolite pathways were followed: (1) ${ }^{13} \mathrm{C}_{3}$-lactate $(m+3$ form $)$ that represents the endpoint of aerobic glycolysis; (2) intermediates of TCA (tricarboxylic acid) cycle (from ${ }^{13} \mathrm{C}_{2}$-citrate to ${ }^{13} \mathrm{C}_{2}$-malate) that represents the mitochondrial OXPHOS pathway and (3) ${ }^{13} \mathrm{C}_{3}$-alanine and ${ }^{13} \mathrm{C}_{3}$-aspartate that indicate other glucose-derived pathways. As shown in Fig. 3, the level of glucose-derived ${ }^{13} \mathrm{C}_{3}$-lactate was significantly raised in ATM-KO C4-2 cells, whereas the levels of glucosederived intermediates of TCA cycle $\left({ }^{13} \mathrm{C}_{2}\right.$-citrate/isocitrate, ${ }^{13} \mathrm{C}_{2}$ - $\alpha$-ketoglutarate, ${ }^{13} \mathrm{C}_{2}$-succinate, ${ }^{13} \mathrm{C}_{2}$-fumarate and ${ }^{13} \mathrm{C}_{2}$-malate) were all reduced in ATM-KO C4-2 cells,

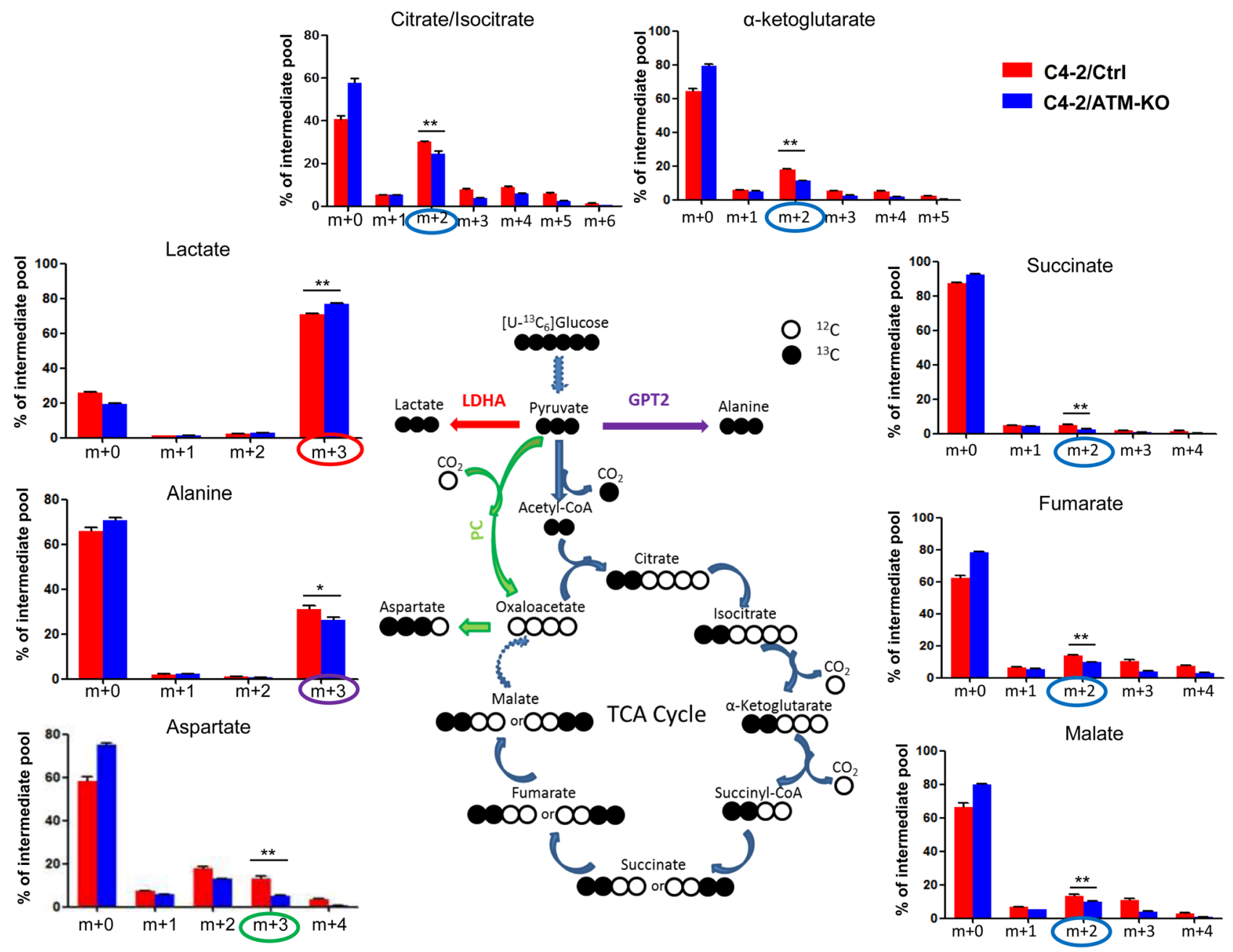

Figure 3

ATM deficiency detours the glucose flux in CRPC cells. C4-2/Ctrl and C4-2/ATM-KO cells were grown in uniformly ${ }^{13} \mathrm{C}-$-labeled glucose medium. The incorporation of ${ }^{13} \mathrm{C}$ atoms from ${ }^{13} \mathrm{C}_{6}$-Glc in to citrate/isocitrate, $\alpha$-ketoglutarate $(\alpha-K G)$, succinate, fumarate, malate, lactate, alanine and aspartate are denoted as $m+n$, where $n$ is the number of ${ }^{13} \mathrm{C}$ atoms. Blue, red, purple and green circles all indicate that ${ }^{13} \mathrm{C}$ atoms are derived from ${ }^{13} \mathrm{C}_{6}-\mathrm{Glc}$ but through different pathways. A scheme describing glucose flows is shown in the middle of the figure. $\mathrm{CO}_{2}$ indicates where carbon dioxide is released; GPT2, glutamate-pyruvate transaminase; LDHA, lactate dehydrogenase A; PC, pyruvate carboxylase. All data are depicted as mean \pm s.D. $* P<0.05$ and $* \star P<0.01$ by two-tailed Student's $t$-test. 
suggesting that ATM deficiency shunts the glucose flux to glycolysis from OXPHOS. The augment of the entry of glucose to glycolysis is further supported by the observation that the production of glucose-derived ${ }^{13} \mathrm{C}_{3}$-alanine and ${ }^{13} \mathrm{C}_{3}$-aspartate was attenuated in C4-2/ATM-KO cells (Fig. 3). A similar result was found in CWRR1 cells as well wherein the ${ }^{13} \mathrm{C}_{3}$-lactate level was increased in CWRR1/ATM-KO cells while the concentrations of other representative glucose-derived metabolites were decreased in CWRR1/ATM-KO cells (Supplementary Fig. 1). Taken together, the glucose tracing results suggest that ATM deficiency further diverts the glucose flux to aerobic glycolytic pathways, i.e. enhances Warburg effect, in CRPC cells.

\section{Upregulation of LDHA contributes to ATM deficiency-induced CRPC progression}

The enhanced Warburg effect as well as the increased production of lactate in ATM-deficient CRPC cells prompted us to determine which enzyme in the glycolysis pathway is regulated by ATM deficiency. We first compared the level of metabolites along the glycolysis pathway from the tracing data and found that only glucosederived lactate, not other intermediate metabolites, was significantly increased in C4-2/ATM-KO cells (Fig. 4A), suggesting that the enzyme LDHA, that is responsible for converting pyruvate to lactate, might be upregulated by ATM deficiency. Actually, we examined the mRNA level of LDHA as well as other enzymes along the glycolysis pathway by RT-qPCR in the two cell lines. Only LDHA was dramatically upregulated in C4-2/ATM-KO cells (Fig. 4B), and this upregulation is further supported by the western blot showing three-fold increase of LDHA protein expression in ATM-KO cells (Fig. 4C). To further confirm the upregulation of LDHA by ATM loss, we treated C4-2 cells with ATM inhibitor KU-60019 and found that the expression of LDHA increased after 72-h treatment of cells with KU-60019 (Supplementary Fig. 2).

The upregulation of LDHA by ATM deficiency facilitated the conversion of glucose-derived pyruvate to lactate (Fig. 4A), which is also expected to decrease the entry of pyruvate to mitochondria for OXPHOS and thereby decrease the production of mitochondrial ROS. We therefore determined the ROS level in C4-2/Ctrl and C4-2/ATM-KO cells by DCFDA (5-(and-6)-carboxy-2', 7 '-dichlorodihydrofluorescein diacetate) fluorescence flow cytometry. As expected, we found that C4-2/ATM-KO cells produced much less ROS than C4-2/Ctrl cells (Fig. 4D), indicating that the cellular oxidative stress is much less in C4-2/ATM-KO cells and consistent with its increased proliferation capacity. Reduction of LDHA is then expected to divert the pyruvate to mitochondria, increase the production of ROS and thereby induce the cell death (Le et al. 2010). We used siRNA to knockdown LDHA expression in both C4-2/Ctrl and C4-2/ATM-KO cells as well as CWRR1/Ctrl and CWRR1/ATM-KO cells (Fig. 4E and Supplementary Fig. 3A) and found that reduction of LDHA by siRNA significantly induced more ROS in C4-2/ATM-KO and CWRR1/ATM-KO cells than C4-2/Ctrl and CWRR1/Ctrl cells (Fig. 4F and Supplementary Fig. 3B) and as a consequence remarkably inhibited cell growth of C4-2/ATM-KO and CWRR1/ATM-KO cells but only caused a marginal inhibition on C4-2/Ctrl and CWRR1/Ctrl cell growth (Fig. 4G and Supplementary Fig. 3C).

\section{LDHA inhibitor FX11 suppresses ATM-deficient CRPC cells growth}

Since inhibition of LDHA by siRNA suppressed the C4-2/ATM-KO cell growth by inducing oxidative stress, we next sought to determine whether LDHA inhibitor has the same inhibitory effect on ATM-deficient CRPC cells that may document a new therapeutic target for this subset of CRPC patients. We selected a small-molecule FX11, which has been shown to preferentially inhibit LDHA and suppress tumor progression (Le et al. 2010). First, we examined the $\mathrm{IC}_{50}$ of FX11 on the two C4-2 cell lines and found that C4-2/ATM-KO cells were more sensitive to the inhibitor than C4-2/Ctrl cells $\left(\mathrm{IC}_{50}=2.52 \mu \mathrm{M}\right.$ for C4-2/ATM-KO vs $\mathrm{IC}_{50}=13.87 \mu \mathrm{M}$ for C4-2/Ctrl) (Fig. 5A). Then, we examined the lactate production after cells being treated with FX11. An obvious reduction of lactate secretion was observed for both C4-2/Ctrl and C4-2/ATM-KO cells, but FX11 treatment resulted in a further significant reduction of lactate production in C4-2/ATM-KO cells (Fig. 5B), manifesting the efficacy of this LDHA inhibitor on ATM-deficient CRPC cells. To determine the effect of FX11 on cell biological functions, we measured the ROS level and cell growth after cells being treated with FX11. Similar to the effect of siLDHA, FX11 treatment led to more ROS production in C4-2/ATM-KO cells and consequently induced more cell death on C4-2/ATM-KO cells (Fig. 5C and D). Additionally, we employed FX11 in CWRR1 cells as well and found the similar results shown in C4-2 cells (Supplementary Fig. 4). To confirm that FX11 also has the efficacy on the ATMdeficient xenograft tumors in vivo, we established the C4-2 xenograft tumors in SCID/NSG mice and intraperitoneally injected either FX11 or vehicle every other day after 
A

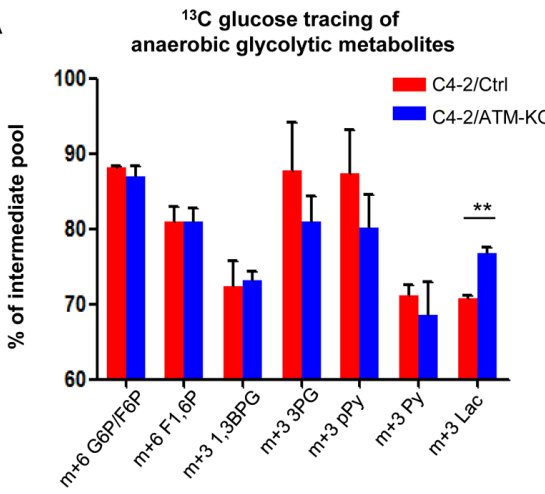

E

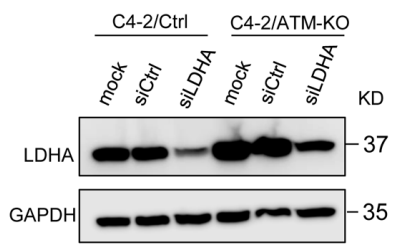

F

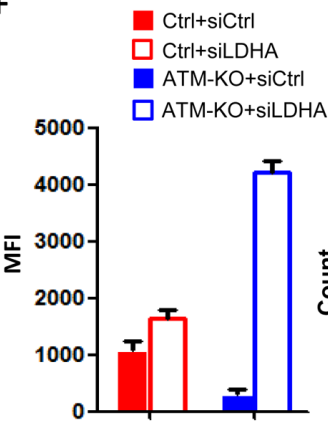

C4-2

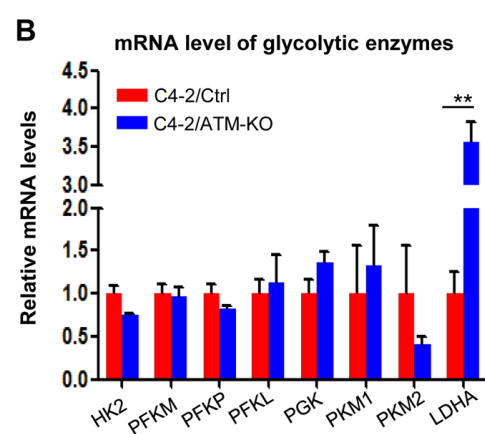

C

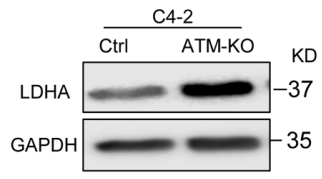

D

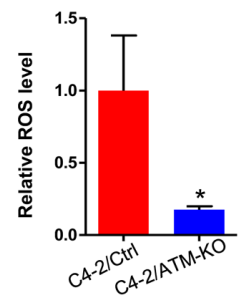

G

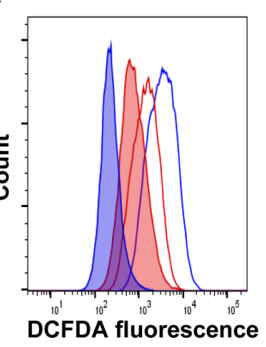

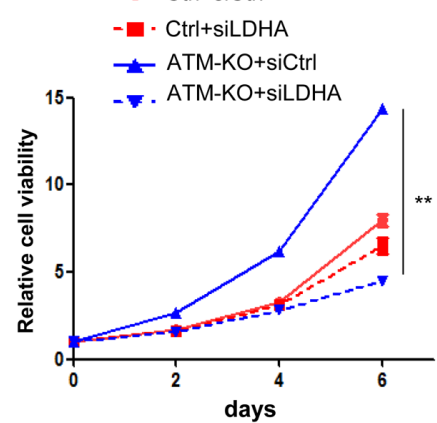

\section{Figure 4}

ATM deficiency upregulates LDHA to promote CRPC progression. (A) ${ }^{13} \mathrm{C}$-isotopomer tracing analysis of glucose-derived glycolytic metabolites in C4-2/Ctrl and C4-2/ATM-KO cells ( $n=3$ ). 1,3BPG, 1,3-bisphosphoglycerate; 3PG, 3-phosphoglycerate; F1,6 P, fructose 1,6-bisphosphate; F6P, fructose 6-phosphate; G6P, glucose 6-phosphate; Lac, lactate; pPy, phosphoenolpyruvate; Py, pyruvate. (B) RT-qPCR to determine the mRNA levels of glycolytic enzymes. Data are normalized to the control group ( $n=3$ independent experiments). HK, hexokinase; LDHA, lactate dehydrogenase A; PFK, phosphofructokinase; PGK, phosphoglycerate kinase; PK, pyruvate kinase. (C) Western blot to determine LDHA protein expression. GAPDH was used as a loading control. (D) Relative level of intracellular ROS. Results are normalized to control group ( $n=3$ independent experiments). (E) C4-2/Ctrl and C4-2/ATM-KO cells were transfected with siRNAs targeting either a scramble sequence (siCtrl) or LDHA (siLDHA). Western blot confirmed knockdown of LDHA expression by using siRNAs. GAPDH was used as a loading control. (F) Intracellular ROS production was detected with DCFDA fluorescence and monitored by flow cytometry at $24 \mathrm{~h}$ post-transfection with siCtrl or siLDHA. Left: median fluorescent intensity. Right: the representative image of histograms of ROS ( $n=3$ independent experiments). (G) Cell viability of C4-2/Ctrl and C4-2/ATM-KO cells transfected with siCtrl and siLDHA was determined by MTS assay at the indicated time points ( $n=3$ replicates). All data are depicted as mean \pm S.D. ${ }^{*} P<0.05$ and $* \star P<0.01$ by two-tailed Student's $t$-test.

tumors reached the size of $200 \mathrm{~mm}^{3}$. As shown in Fig. $5 \mathrm{E}$ and F, FX11 profoundly inhibited tumor growth of C4-2/ATM-KO xenografts but had a marginal insignificant inhibition on C4-2/Ctrl xenograft tumors, in a similar manner to the in vitro results (Fig. 5D).

\section{ATM deficiency upregulates LDHA expression by enhancing c-Myc level}

Next, we sought to explore the potential mechanism how LDHA is regulated by ATM. The link between LDHA and c-Myc has been well established and LDHA is identified as a c-Myc-responsive gene (Lewis et al. 1997, Shim et al. 1997, He et al. 2015). In addition, it has been shown that c-Myc is upregulated by ATM inactivation in response to replication stress (Aird et al. 2015). Hence, we wanted to know whether the upregulation of LDHA by ATM loss is mediated by c-Myc. First, we examined the c-Myc protein level in both C4-2/Ctrl and C4-2/ATM-KO cells and found that c-Myc protein level was dramatically increased in ATM-deficient C4-2 cells (Fig. 6A). Then, we silenced c-Myc by siRNAs and found that knockdown of c-Myc resulted in reduced LDHA expression at mRNA and protein levels in both C4-2/Ctrl and C4-2/ATM-KO cells (Fig. 6B and C), further confirming the ATM regulation of LDHA through c-Myc.

\section{Discussion}

Collectively, we demonstrated in this study that ATM deficiency promoted the cell proliferation of CRPC cells by enhancing Warburg effect. ATM deficiency induced LDHA upregulation that shunted the glucose-derived pyruvate into aerobic glycolysis to generate more lactate 
A

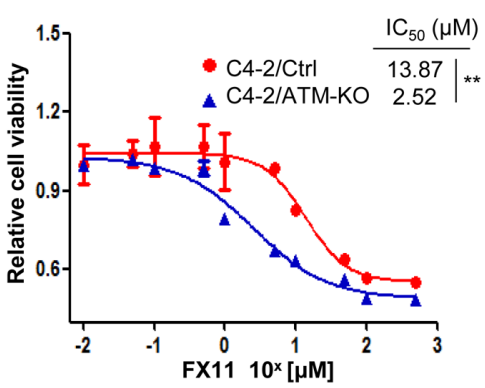

C

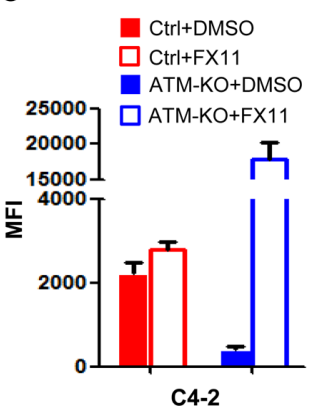

B

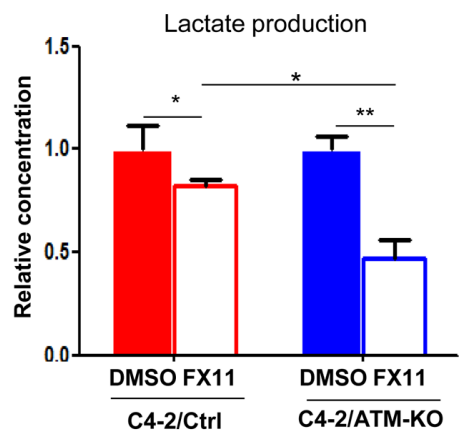

E

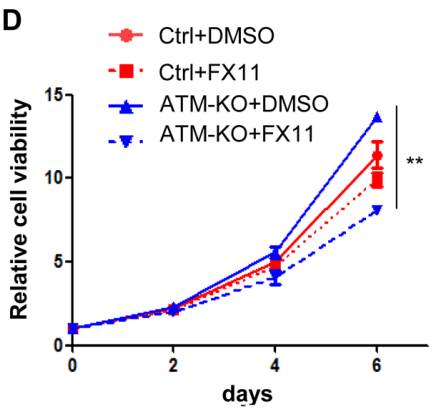

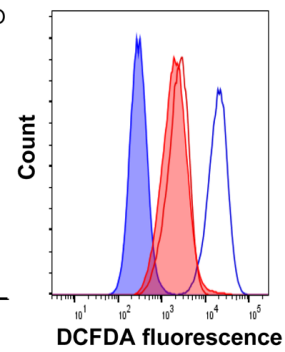

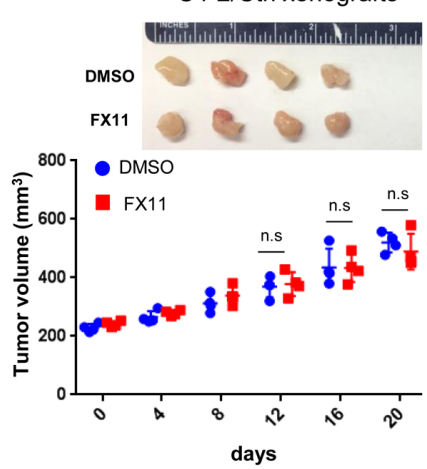

\section{Figure 5}

LDHA inhibitor FX11 suppresses ATM-deficient CRPC cell growth. (A) IC 50 values of FX11 were measured on C4-2/Ctrl and C4-2/ATM-KO cells treated with a series concentration of FX11 for $72 \mathrm{~h}$. (B) Lactate production of C4-2/Ctrl and C4-2/ATM-KO cells treated with either DMSO or FX11 for $72 \mathrm{~h}$. Results are normalized to DMSO group and set as ' 1 '. (C) Intracellular ROS production was detected with DCFDA fluorescence and monitored by flow cytometry at $72 \mathrm{~h}$ post-treatment with either DMSO or $3 \mu \mathrm{M}$ FX11. Left: median fluorescent intensity. Right: the representative image of histograms of ROS ( $n=3$ independent experiments). (D) Cell viability of C4-2/Ctrl and C4-2/ATM-KO cells treated with either DMSO or FX11 was determined by MTS assay at the indicated time points ( $n=3$ replicates). (E and F) Tumor growth of C4-2/Ctrl and C4-2/ATM-KO xenografts treated intraperitoneally with either DMSO or FX11 when tumor reached $\sim 200 \mathrm{~mm}^{3}$. Tumor volumes were measured every 4 days. Top: Representative tumor images in each group. Bottom: The growth curve of xenograft tumors after treatment of vehicle (DMSO) or FX11. All data are depicted as mean \pm S.D. ${ }^{*} P<0.05$ and ${ }^{* *} P<.01$ by two-tailed Student's t-test.

and simultaneously reduced the entry of pyruvate to mitochondria and therefore produced less ROS. Both increased lactate and reduced ROS cooperated to promote the progression of CRPC (Fig. 6D). Further, we showed that inhibition of LDHA by siRNA or inhibitor FX11 decreased the lactate production and increased ROS level in ATM-deficient CRPC cells and therefore potentiated the cell death of ATM-deficient CRPC cells (Fig. 6D). Additionally, we demonstrated that c-Myc mediates the upregulation of LDHA by ATM loss (Fig. 6D). These findings for the first time reveal a novel non-DNA repair mechanism whereby ATM mutations contribute to the CRPC progression and suggest an alternative therapeutic target for the treatment of ATM mutant CRPC tumors in addition to PARP inhibitors.

Although the clinical impact of ATM mutation (and BRAC1/2 mutation) on CRPC patients' response to AR-targeted therapies remains debated (Annala et al.
2017, Annala et al. 2018, Antonarakis et al. 2018, Hussain et al. 2018), the increased prevalence of ATM mutation in therapeutic resistant mCRPC patients and the worse prognosis of ATM mutant prostate cancer patients implicate the important role of ATM deficiency in the disease progression of prostate cancer and therapeutic resistance development. ATM and BRCA2 are known to play a critical role in homologous recombination repair (HRR) of DNA double-strand breaks. The clinical observation that ATM-mutated and BRCA2-mutated tumors are enriched in mCRPC suggests that ATM deficiency and/or BRCA2 deficiency should re-establish other pathways to drive the disease progression irrespective of defective HRR DNA repair. Actually, germline BRCA2 mutant prostate tumors have a mutational profile that more closely resembles metastatic and aggressive prostate tumors and deregulates MED12L/MED12 pathway (Taylor et al. 2017). Unfortunately, the genomic and epi-genomic profiles of 
A

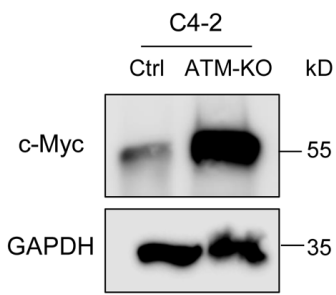

D

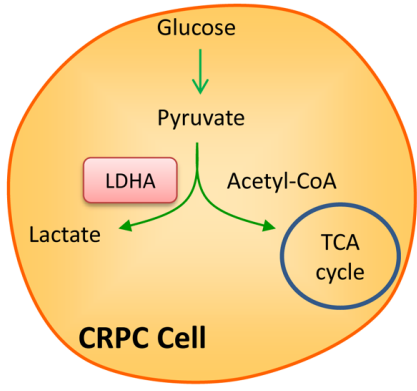

B

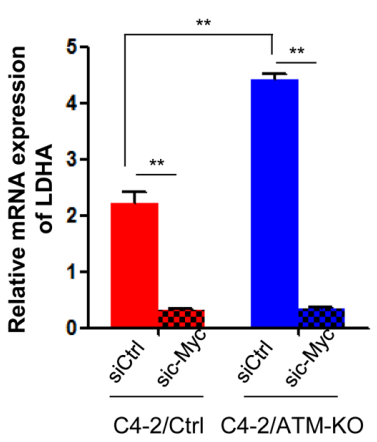

C

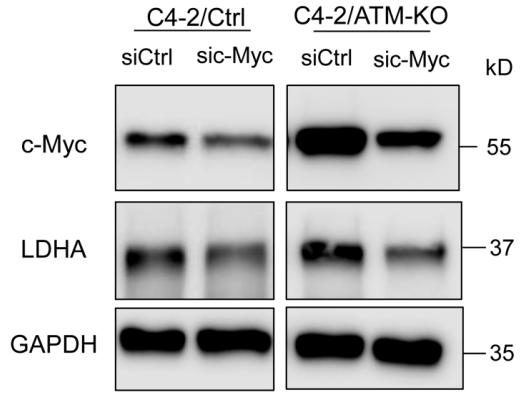

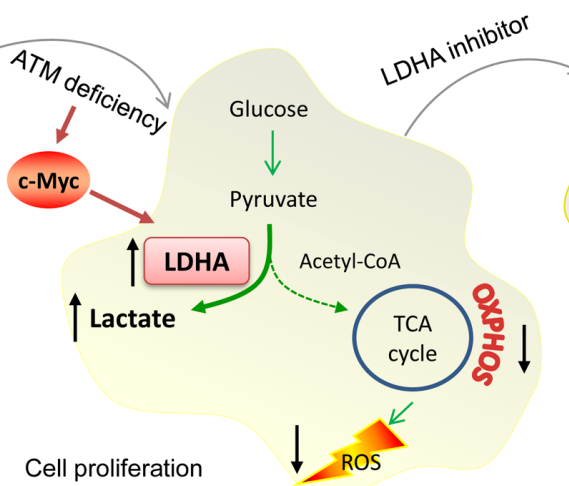

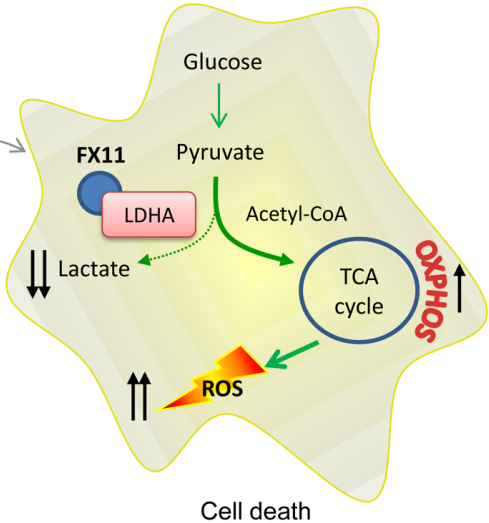

Cell death

Figure 6

ATM deficiency upregulates LDHA expression via enhancing c-Myc level. (A) Western blot to determine c-Myc protein expression in C4-2/Ctrl and C4-2/ ATM-KO cells. GAPDH was used as a loading control. (B and C) C4-2/Ctrl and C4-2/ATM-KO cells were transfected with siRNAs targeting either a scramble sequence (siCtrl) or c-Myc (sic-Myc). RT-qPCR confirmed that LDHA mRNA was downregulated after c-Myc knockdown in both C4-2/Ctrl and C4-2/ATM-KO cells (B) and Western blot confirmed knockdown of c-Myc expression by siRNAs and the subsequent downregulation of LDHA protein expression (C). GAPDH was used as a loading control. (D) A working model of up-regulated LDHA contributing to the progression of ATM-deficient CRPC.

ATM-mutant prostate tumors are still not available. Here we used CRPC cell and xenograft models to demonstrate that ATM deficiency resulted in CRPC progression by enhancing Warburg effect, which may shed new light on the issue of how ATM mutation driving prostate cancer progression. Transgenic or prostate conditional Atmknockout mouse model may help to solve this issue and debate.

ATM can modulate carbon metabolism through many pathways such as glycolysis and pentose phosphate pathway (PPP) (Cosentino et al. 2011, Kruger \& Ralser 2011). In regard to glycolysis, ATM has been shown to transcriptionally modulate glucose transporter genes SLC2A1 and SLC2A4, which encode for GLUT1 and GLUT4, respectively (Schwartzenberg-Bar-Yoseph et al. 2004). Here, we demonstrated that ATM deficiency or inhibition led to upregulation of LDHA in both mRNA and protein levels (Fig. 4 and Supplementary Fig. 2) and the potential underlying mechanism might be mediated by transcription factor c-Myc (Fig. 6). Interestingly, these findings are quite consistent with a recent study that ATM inactivation enhances glucose consumption by upregulating c-Myc expression (Aird et al. 2015) and other previous studies that c-Myc is positively correlated with LDHA expression in lymphoid cells and pancreatic cancer cells (Lewis et al. 1997, Shim et al. 1997, He et al. 2015). LDHA is overexpressed in many cancers and high LDHA levels frequently correlate with poor prognosis and survival (Girgis et al. 2014). More importantly, based on a most recent study, LDHA has been implicated as significant clinical biomarker since its genomic abnormalities would link to high pretreatment LDH level and poor response to docetaxel in mCRPC (Hiew et al. 2018). Furthermore, $64.3 \%$ of the patients with high LDH level were determined to have DNA repair gene mutations such as BRCA1/2 and ATM by next-generation sequencing (Hiew et al. 2018). Inhibition of LDHA in multiple tumor models decreased tumor growth, emphasizing the importance of LDHA in tumor progression (Le et al. 2010, Xie et al. 2014). Lactate, which has been merely considered as a metabolic by-product for decades, is now recognized as a primary source of carbon for energy production (Hui et al. 2017) 
as well as a multi-functional metabolite that can promote cancer growth (San-Millan \& Brooks 2017). Upregulating LDHA expression also reduces the carbon flow from pyruvate into the TCA cycle, thereby minimizing ROS that is normally generated as byproducts of OXPHOS (Newington et al. 2012). Our data revealed that C4-2/ ATM-KO cells had less ROS and produced more lactate, both may cooperate together to promote CRPC cells growth (Fig. 4). Interestingly, another study reported that inhibiting ATM by ATM inhibitor KU-55933 stimulates the glycolysis pathway while reducing mitochondrial membrane potential and coupled respiration (Zakikhani et al. 2012), which is corroborating with our findings in ATM-depleted CRPC cells.

PARP inhibitors, based on the synthetic lethality strategy, have been shown effective to treat BRCA1/2 mutation-bearing ovarian cancer and breast cancer (George et al. 2016). Mateo et al. also demonstrated that PARP inhibitor Olaparib as a monotherapy can lead to a high response rate in patients who had defects in DNA repair genes (mostly BRCA2 and ATM) (Mateo et al. 2015), which paves the way to use PARP inhibitor for the treatment of mCRPC patients with DRG mutations. However, PARP inhibitor resistance have been shown to develop in ovarian and breast cancer and the underlying resistant mechanisms are mostly related to the restoration of HRR repair capacity in these DRG-mutant tumors (Lord et al. 2015). In this study, we demonstrated that ATM deficiency promoted CRPC tumor growth through its non-DNA repair function and our data indicated that ATM-deficient CRPC cells were more sensitive to LDHA inhibitors, strongly suggesting an alternative therapeutic option for ATM-mutant CRPC tumors by targeting LDHA, which may also be effective for the PAPR inhibitorresistant CRPC tumors.

\section{Supplementary data}

This is linked to the online version of the paper at https://doi.org/10.1530/ ERC-18-0196.

\section{Declaration of interest}

The authors declare that there is no conflict of interest that could be perceived as prejudicing the impartiality of the research reported.

\section{Funding}

This study was supported by NIH grants R01 CA172603 (J Huang), R01 CA205001 (J Huang) and National Natural Science Foundation of China (\#81630019).

\section{Acknowledgements}

The authors thank Drs Yanjing Li, Yu Yin and Yan Chang for their technical help; Dr Yen-Nien Liu for his comments. They thank Drs Juan Liu and Jason Locasale for their expertise in LC-MS analysis and tracing.

\section{References}

Abida W, Armenia J, Gopalan A, Brennan R, Walsh M, Barron D, Danila D, Rathkopf D, Morris M, Slovin S, et al. 2017 Prospective genomic profiling of prostate cancer across disease states reveals germline and somatic alterations that may affect clinical decision making. JCO Precision Oncology 2017 [epub]. (https://doi. org/10.1200/PO.17.00029)

Aird KM, Worth AJ, Snyder NW, Lee JV, Sivanand S, Liu Q, Blair IA, Wellen KE \& Zhang R 2015 ATM couples replication stress and metabolic reprogramming during cellular senescence. Cell Reports $\mathbf{1 1}$ 893-901. (https://doi.org/10.1016/j.celrep.2015.04.014)

Ambrose M \& Gatti RA 2013 Pathogenesis of ataxia-telangiectasia: the next generation of ATM functions. Blood 121 4036-4045. (https:// doi.org/10.1182/blood-2012-09-456897)

Ambrose M, Goldstine JV \& Gatti RA 2007 Intrinsic mitochondrial dysfunction in ATM-deficient lymphoblastoid cells. Human Molecular Genetics 16 2154-2164. (https://doi.org/10.1093/hmg/ddm166)

Annala M, Struss WJ, Warner EW, Beja K, Vandekerkhove G, Wong A, Khalaf D, Seppälä I-L, So A \& Lo G 2017 Treatment outcomes and tumor loss of heterozygosity in germline DNA repair-deficient prostate cancer. European Urology 72 34-42. (https://doi. org/10.1016/j.eururo.2017.02.023)

Annala M, Vandekerkhove G, Khalaf D, Taavitsainen S, Beja K, Warner EW, Sunderland K, Kollmannsberger C, Eigl BJ \& Finch D 2018 Circulating tumor DNA genomics correlate with resistance to abiraterone and enzalutamide in prostate cancer. Cancer Discovery 8 444-457. (https://doi.org/10.1158/2159-8290.CD-17-0937)

Antonarakis ES, Lu C, Luber B, Liang C, Wang H, Chen Y, Silberstein JL, Piana D, Lai Z, Chen Y, et al. 2018 Germline DNA-repair gene mutations and outcomes in men with metastatic castration-resistant prostate cancer receiving first-line abiraterone and enzalutamide. European Urology 74 218-225. (https://doi.org/10.1016/j. eururo.2018.01.035)

Beltran H, Yelensky R, Frampton GM, Park K, Downing SR, MacDonald TY, Jarosz M, Lipson D, Tagawa ST \& Nanus DM 2013 Targeted next-generation sequencing of advanced prostate cancer identifies potential therapeutic targets and disease heterogeneity. European Urology 63 920-926. (https://doi.org/10.1016/j. eururo.2012.08.053)

Cairns RA, Harris IS \& Mak TW 2011 Regulation of cancer cell metabolism. Nature Reviews Cancer 11 85-95. (https://doi. org/10.1038/nrc2981)

Choi M, Kipps T \& Kurzrock R 2016 ATM mutations in cancer: therapeutic implications. Molecular Cancer Therapeutics $\mathbf{1 5}$ 1781-1791. (https://doi.org/10.1158/1535-7163.MCT-15-0945)

Cosentino C, Grieco D \& Costanzo V 2011 ATM activates the pentose phosphate pathway promoting anti-oxidant defence and DNA repair. EMBO Journal 30 546-555. (https://doi.org/10.1038/emboj.2010.330)

Fraser M, Sabelnykova VY, Yamaguchi TN, Heisler LE, Livingstone J, Huang V, Shiah YJ, Yousif F, Lin X, Masella AP, et al. 2017 Genomic hallmarks of localized, non-indolent prostate cancer. Nature $\mathbf{5 4 1}$ 359-364. (https://doi.org/10.1038/nature20788)

George A, Kaye S \& Banerjee S 2016 Delivering widespread BRCA testing and PARP inhibition to patients with ovarian cancer. Nature Reviews Clinical Oncology 14 284-296. (https://doi.org/10.1038/ nrclinonc.2016.191)

Girgis H, Masui O, White NM, Scorilas A, Rotondo F, Seivwright A, Gabril M, Filter ER, Girgis AH \& Bjarnason GA 2014 Lactate https://erc.bioscientifica.com

https://doi.org/10.1530/ERC-18-0196 (c) 2019 Society for Endocrinology Published by Bioscientifica Ltd. Printed in Great Britain 
dehydrogenase A is a potential prognostic marker in clear cell renal cell carcinoma. Molecular Cancer 13 101. (https://doi. org/10.1186/1476-4598-13-101)

He TL, Zhang YJ, Jiang H, Li XH, Zhu H \& Zheng KL 2015 The c-MycLDHA axis positively regulates aerobic glycolysis and promotes tumor progression in pancreatic cancer. Medical Oncology 32187 (https://doi.org/10.1007/s12032-015-0633-8)

Hiew K, Hart CA, Ali A, Elliott T, Ramani V, Sangar V, Lau M, Maddineni S, Brown M \& Clarke N 2018 Primary mutational landscape linked with pre-docetaxel lactate dehydrogenase levels predicts docetaxel response in metastatic castrate-resistant prostate cancer. European Urology Focus [epub]. (https://doi.org/10.1016/j. euf.2018.04.006)

Hu H \& Gatti RA 2008 New approaches to treatment of primary immunodeficiencies: fixing mutations with chemicals. Current Opinion in Allergy and Clinical Immunology 8 540-546. (https://doi. org/10.1097/ACI.0b013e328314b63b)

Hui S, Ghergurovich JM, Morscher RJ, Jang C, Teng X, Lu W, Esparza LA, Reya T, Zhan L \& Guo JY 2017 Glucose feeds the TCA cycle via circulating lactate. Nature 551 115. (https://doi. org/10.1038/nature24057)

Hussain M, Daignault-Newton S, Twardowski PW, Albany C, Stein MN, Kunju LP, Siddiqui J, Wu YM, Robinson D, Lonigro RJ, et al. 2018 Targeting androgen receptor and DNA repair in metastatic castration-resistant prostate cancer: results from NCI 9012. Journal of Clinical Oncology 36 991-999. (https://doi.org/10.1200/ JCO.2017.75.7310)

Kruger A \& Ralser M 2011 ATM is a redox sensor linking genome stability and carbon metabolism. Science Signaling 4 pe17.

Le A, Cooper CR, Gouw AM, Dinavahi R, Maitra A, Deck LM, Royer RE, Vander Jagt DL, Semenza GL \& Dang CV 2010 Inhibition of lactate dehydrogenase A induces oxidative stress and inhibits tumor progression. PNAS 107 2037-2042. (https://doi.org/10.1073/ pnas.0914433107)

Lee P, Martin NT, Nakamura K, Azghadi S, Amiri M, Ben-David U, Perlman S, Gatti RA, Hu H \& Lowry WE 2013 SMRT compounds abrogate cellular phenotypes of ataxia telangiectasia in neural derivatives of patient-specific hiPSCs. Nature Communications 41824. (https://doi.org/10.1038/ncomms2824)

Lewis BC, Shim H, Li Q, Wu CS, Lee LA, Maity A \& Dang CV 1997 Identification of putative c-Myc-responsive genes: characterization of rcl, a novel growth-related gene. Molecular and Cellular Biology 17 4967-4978. (https://doi.org/10.1128/MCB.17.9.4967)

Liberti MV \& Locasale JW 2016 The Warburg effect: how does it benefit cancer cells? Trends in Biochemical Sciences 41 211-218. (https://doi. org/10.1016/j.tibs.2015.12.001)

Lord CJ, Tutt AN \& Ashworth A 2015 Synthetic lethality and cancer therapy: lessons learned from the development of PARP inhibitors. Annual Review of Medicine 66 455-470. (https://doi.org/10.1146/ annurev-med-050913-022545)

Mateo J, Carreira S, Sandhu S, Miranda S, Mossop H, Perez-Lopez R, Nava Rodrigues D, Robinson D, Omlin A \& Tunariu N 2015 DNArepair defects and olaparib in metastatic prostate cancer. New England Journal of Medicine 373 1697-1708. (https://doi.org/10.1056/ NEJMoa1506859)

Na R, Zheng SL, Han M, Yu H, Jiang D, Shah S, Ewing CM, Zhang L, Novakovic K \& Petkewicz J 2017 Germline mutations in ATM and BRCA1/2 distinguish risk for lethal and indolent prostate cancer and are associated with early age at death. European Urology 71 740-747. (https://doi.org/10.1016/j.eururo.2016.11.033)

Newington JT, Rappon T, Albers S, Wong DY, Rylett RJ \& Cumming RC 2012 Overexpression of pyruvate dehydrogenase kinase 1 and lactate dehydrogenase $\mathrm{A}$ in nerve cells confers resistance to amyloid $\beta$ and other toxins by decreasing mitochondrial respiration and reactive oxygen species production. Journal of Biological Chemistry $\mathbf{2 8 7}$ 37245-37258. (https://doi.org/10.1074/jbc.M112.366195)

Pan M, Reid MA, Lowman XH, Kulkarni RP, Tran TQ, Liu X, Yang Y, Hernandez-Davies JE, Rosales KK \& Li H 2016 Regional glutamine deficiency in tumours promotes dedifferentiation through inhibition of histone demethylation. Nature Cell Biology 18 1090. (https://doi. org/10.1038/ncb3410)

Park JW, Lee JK, Witte ON \& Huang J 2017 FOXA2 is a sensitive and specific marker for small cell neuroendocrine carcinoma of the prostate. Modern Pathology 30 1262. (https://doi.org/10.1038/ modpathol.2017.44)

Pavlova NN \& Thompson CB 2016 The emerging hallmarks of cancer metabolism. Cell Metabolism 23 27-47. (https://doi.org/10.1016/j. cmet.2015.12.006)

Robinson D, Van Allen EM, Wu Y-M, Schultz N, Lonigro RJ, Mosquera JM, Montgomery B, Taplin M-E, Pritchard CC \& Attard G 2015 Integrative clinical genomics of advanced prostate cancer. Cell $\mathbf{1 6 1}$ 1215-1228. (https://doi.org/10.1016/j.cell.2015.05.001)

San-Millan I \& Brooks GA 2017 Reexamining cancer metabolism: lactate production for carcinogenesis could be the purpose and explanation of the Warburg Effect. Carcinogenesis 38 119-133.

Schwartzenberg-Bar-Yoseph F, Armoni M \& Karnieli E 2004 The tumor suppressor p53 down-regulates glucose transporters GLUT1 and GLUT4 gene expression. Cancer Research 64 2627-2633. (https://doi. org/10.1158/0008-5472.CAN-03-0846)

Shiloh Y 2014 ATM: expanding roles as a chief guardian of genome stability. Experimental Cell Research 329 154-161. (https://doi. org/10.1016/j.yexcr.2014.09.002)

Shiloh Y \& Ziv Y 2013 The ATM protein kinase: regulating the cellular response to genotoxic stress, and more. Nature Reviews Molecular Cell Biology 14 197-210. (https://doi.org/10.1038/nrm3546)

Shim H, Dolde C, Lewis BC, Wu CS, Dang G, Jungmann RA, DallaFavera R \& Dang CV 1997 c-Myc transactivation of LDH-A: implications for tumor metabolism and growth. PNAS 94 6658-6663. (https://doi.org/10.1073/pnas.94.13.6658)

Taylor RA, Fraser M, Livingstone J, Espiritu SM, Thorne H, Huang V, Lo W, Shiah YJ, Yamaguchi TN, Sliwinski A, et al. 2017 Germline BRCA2 mutations drive prostate cancers with distinct evolutionary trajectories. Nature Communications 8 13671. (https://doi. org/10.1038/ncomms13671)

Warburg O 1956 On the origin of cancer cells. Science 123 309-314. (https://doi.org/10.1126/science.123.3191.309)

Xie H, Hanai J-I, Ren J-G, Kats L, Burgess K, Bhargava P, Signoretti S, Billiard J, Duffy KJ \& Grant A 2014 Targeting lactate dehydrogenase-a inhibits tumorigenesis and tumor progression in mouse models of lung cancer and impacts tumor-initiating cells. Cell Metabolism 19 795-809. (https://doi.org/10.1016/j.cmet.2014.03.003)

Zakikhani M, Bazile M, Hashemi S, Javeshghani S, Avizonis D, St Pierre J \& Pollak MN 2012 Alterations in cellular energy metabolism associated with the antiproliferative effects of the ATM inhibitor KU-55933 and with metformin. PLOS ONE 7 e49513. (https://doi. org/10.1371/journal.pone.0049513)

Received in final form 2 August 2018

Accepted 7 August 2018 https://erc.bioscientifica.com https://doi.org/10.1530/ERC-18-0196
(C) 2019 Society for Endocrinology Published by Bioscientifica Ltd. Printed in Great Britain 